\title{
NEW QUARTZ CRYSTAL OSCILLATORS USING THE CURRENT-FEEDBACK OPERATIONAL AMPLIFIER
}

\author{
MUHAMMAD TAHER ABUELMA'ATTI* \\ and AHMAD AL-GHAZWANI \\ King Fahd University of Petroleum and Minerals, Box 203, \\ Dhahran 31261, Saudi Arabia
}

(Received 7 February 2000; In final form 25 April 2000)

\begin{abstract}
New crystal oscillators using the current-feedback operational-amplifier (CFOA) are presented. Each circuit uses one CFOA, a crystal and, at most, five externally connected passive elements. Experimental results are included.
\end{abstract}

Keywords: Oscillators; Current-feedback operational amplifier

\section{INTRODUCTION}

Quartz crystal oscillators built around various discrete and integratedcircuit active elements are available in the literature [1,2]. Of particular interest here are crystal oscillators built around the second-generation current-conveyor (CCII) [3-5]. Because the CCII minimally degrades the quartz crystal performance, CCII-based crystal oscillators enjoy good frequency stability and are, therefore, very attractive [3].

On the other hand, the current-feedback operational-amplifier (CFOA) is a four-terminal device which is equivalent to a CCII plus an output voltage buffer and, thus, it can easily drive external loads. Moreover, the CFOA enjoys wide bandwidth which is relatively

${ }^{*}$ Corresponding author. 
independent of the closed-loop gain and very high slew rate. Thus, the use of CFOA in realizing crystal oscillators will be attractive.

To the authors knowledge, the use of CFOA is designing crystal oscillators has not yet been reported in the literature. It is, therefore, the purpose of this letter to present a number of CFOA-based crystal oscillators.

\section{PROPOSED CIRCUITS}

Consider the oscillator structures shown in Figure 1. Assuming ideal CFOA with $i_{z}=i_{x}, i_{y}=0, v_{x}=v_{y}, v_{o}=v_{z}$ routine analysis yields the characteristic equation given by

$$
\left(Y_{1}+Y_{2}\right) Y_{4}+Y_{2} Y_{3}=0
$$

for the oscillator structure of Figure 1(a), and

$$
\left(Y_{1}+Y_{2}\right) Y_{4}-Y_{2} Y_{3}=0
$$

for the oscillator structure of Figure 1(b).

Using the crystal equivalent circuit shown in Figure 2, the admittance $Y_{3}$ can be expressed as

$$
Y_{3}=\frac{r+s L_{1}+1 / s C_{1}+1 / s C_{0}}{r / s C_{0}+L_{1} / C_{0}+1 / s^{2} C_{1} C_{0}}
$$

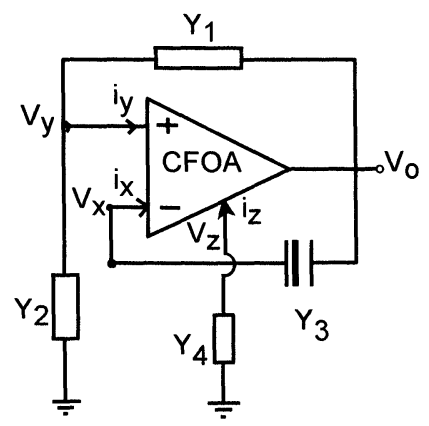

(a)

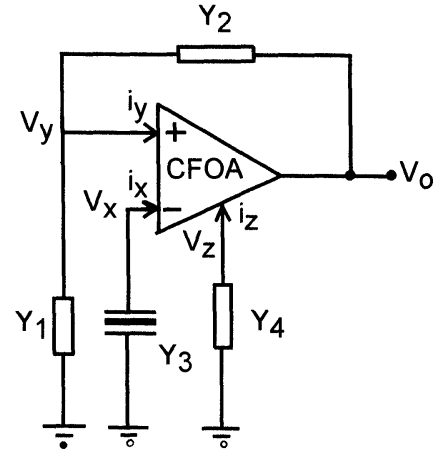

(b)

FIGURE 1 Proposed crystal oscillator structures. 


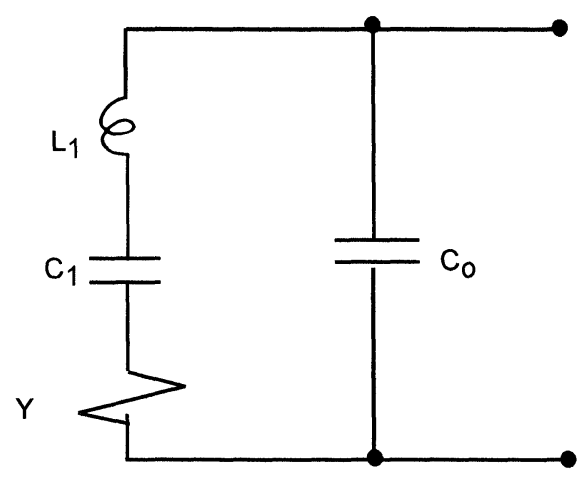

FIGURE 2 Electrical equivalent circuit of quartz crystal.

where $r$ is the crystal internal resistance, $L_{1}$ is the crystal internal inductance, $C_{1}$ is the crystal internal capacitance, and $C_{0}$ is the electrostatic capacitance between the two parallel plates of the crystal.

Combining Eqs. (1) and (2), new CFOA-based crystal oscillators can be obtained, from the oscillator structures of Figure 1, using various combinations of the admittances $Y_{1}, Y_{2}$ and $Y_{4}$. Following are two examples:

Example 1 In Figure $1(\mathrm{a})$, if $Y_{1}=1 / R, Y_{2}=1 / R+s C_{2}$ and $Y_{4}=$ $1 / R+s C_{4}$, then Eq. (1a) reduces to

$$
\begin{aligned}
& s^{4} L_{1} C_{2}\left(\frac{C_{4}}{C_{0}}+1\right)+s^{3}\left(\frac{L_{1}}{R}\left(\frac{C_{2}}{C_{0}}+\frac{2 C_{4}}{C_{0}}+1\right)+C_{2} r\left(\frac{C_{4}}{C_{0}}+1\right)\right) \\
& +s^{2}\left(\frac{C_{2}}{C_{0}}\left(\frac{C_{4}}{C_{1}}+\frac{r}{R}+1\right)+\frac{2 C_{4}}{C_{0}} \frac{r}{R}+2 \frac{L_{1}}{R^{2} C_{0}}+\frac{r}{R}+\frac{C_{2}}{C_{1}}\right) \\
& +s\left(\frac{1}{C_{0} R}\left(\frac{C_{2}}{C_{1}}+\frac{2 C_{4}}{C_{1}}+2 \frac{r}{R}+1\right)+\frac{1}{C_{1} R}\right)+2 \frac{1}{R^{2} C_{1} C_{0}}=0
\end{aligned}
$$

Equation (3) is a fourth-order polynomial in $s$. Following a procedure similar to that described in [6], by disregarding the $s^{4}$ term and substituting for $s^{3}$ the term

$$
s^{3}=-s \omega_{p}^{2}
$$

Equation (3) reduces to a second-order polynomial in $s$. Equating the real and imaginary parts, of this second-order polynomial to zero, 
yields

$$
\omega_{p}^{2}=\frac{\left(1 / C_{0} R\right)\left(\left(C_{2} / C_{1}\right)+2\left(C_{4} / C_{1}\right)+2(r / R)+1\right)+\left(1 / R C_{1}\right)}{\left(L_{1} / R\right)\left(\left(C_{2} / C_{0}\right)+2\left(C_{4} / C_{0}\right)+1\right)+C_{2} r\left(\left(C_{4} / C_{0}\right)+1\right)}
$$

and

$\omega_{n}^{2}=\frac{2 /\left(R^{2} C_{1} C_{0}\right)}{\left(C_{2} / C_{0}\right)\left(\left(C_{4} / C_{1}\right)+(r / R)+1\right)+2\left(C_{4} / C_{0}\right)(r / R)+2\left(L_{1} / R^{2} C_{0}\right)+\left(C_{2} / C_{1}\right)}$

With $C_{1} \ll C_{0}, r \ll R$, Eqs. (5) and (6) can be approximated by

$$
\omega_{p}^{2} \cong \frac{1}{L_{1} C_{1}}
$$

and

$$
\omega_{n}^{2} \cong \frac{1}{L_{1} C_{1}}
$$

From (7) and (8) it obvious than $\omega_{p}$ and $\omega_{n}$ are approximately equal and thus, the circuit can oscillate at a frequency approximately given by the series-resonance frequency of the crystal.

Example 2 In Figure 1(b), if $Y_{1}=1 / R, Y_{2}=1 / R+s C_{2}$ and $Y_{4}=$ $1 / R+s C_{4}$, then Eq. (1b) reduces to

$$
\begin{aligned}
& s^{4} L_{1} C_{2}\left(\frac{C_{4}}{C_{0}}-1\right)+s^{3}\left(\frac{L_{1}}{R}\left(\frac{C_{2}}{C_{0}}+\frac{2 C_{4}}{C_{0}}-1\right)+C_{2} r\left(\frac{C_{4}}{C_{0}}-1\right)\right) \\
& +s^{2}\left(\frac{C_{2}}{C_{0}}\left(\frac{C_{4}}{C_{1}}+\frac{r}{R}-1\right)+\frac{2 C_{4}}{C_{0}} \frac{r}{R}+2 \frac{L_{1}}{R^{2} C_{0}}-\frac{r}{R}-\frac{C_{2}}{C_{1}}\right) \\
& +s\left(\frac{1}{C_{0} R}\left(\frac{C_{2}}{C_{1}}+\frac{2 C_{4}}{C_{1}}+2 \frac{r}{R}-1\right)-\frac{1}{C_{1} R}\right)+2 \frac{1}{R^{2} C_{1} C_{0}}=0
\end{aligned}
$$

Equation (9) is a fourth-order polynomial in $s$. Following similar procedure as in the previous example, it is easy to, show that

$$
\omega_{p}^{2}=\frac{\left(1 / C_{0} R\right)\left(\left(C_{2} / C_{1}\right)+2\left(C_{4} / C_{1}\right)+2(r / R)-1\right)-\left(1 / R C_{1}\right)}{\left(L_{1} / R\right)\left(\left(C_{2} / C_{0}\right)+2\left(C_{4} / C_{0}\right)-1\right)+C_{2} r\left(\left(C_{4} / C_{0}\right)-1\right)}
$$


and

$\omega_{n}^{2}=\frac{2 /\left(R^{2} C_{1} C_{0}\right)}{\left(C_{2} / C_{0}\right)\left(\left(C_{4} / C_{1}\right)+(r / R)-1\right)+2\left(C_{4} / C_{0}\right)(r / R)+2\left(L_{1} / R^{2} C_{0}\right)-\left(C_{2} / C_{1}\right)}$

With $C_{1} \ll C_{0}, r \ll R$, Eqs. (10) and (11) can be approximated by Eqs. (7) and (8) respectively. Thus, the circuit can oscillate at a frequency approximately equal to the series-resonance frequency of the crystal.

\section{EXPERIMENTAL RESULTS}

The proposed circuits of Figure 1 were tested using the AD844 CFOA and different crystals. A typical output waveform obtained from the circuit of Figure 1(a) with $R=10 \mathrm{k} \Omega$ and $20 \mathrm{MHz}$ crystal is shown in Figure 3. Only three externally connected equal resistances were used. No external capacitances were connected. In fact the parasitic

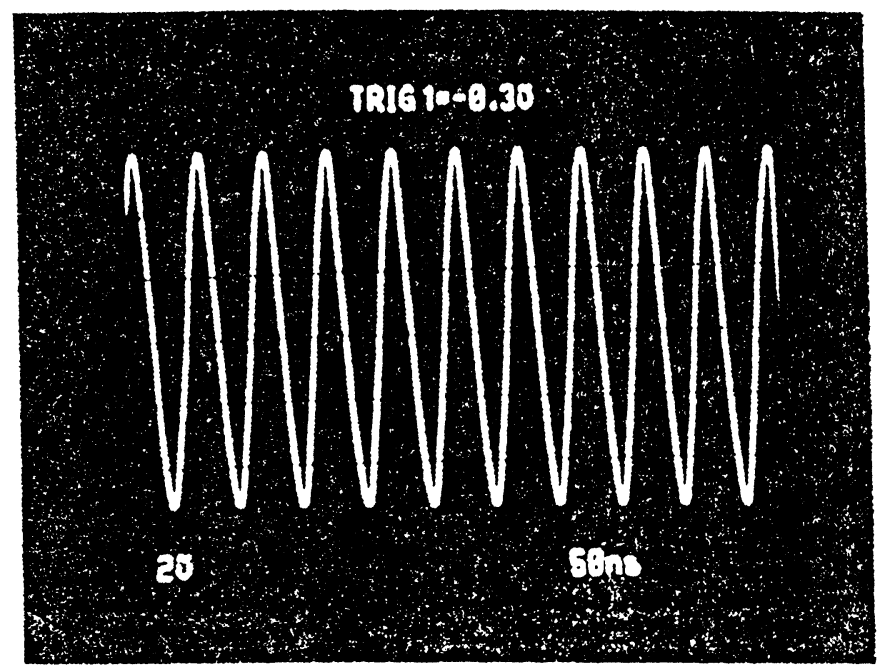

FIGURE 3 Typical output waveform obtained from the osciallator structure of Figure 1(a) with: $Y_{1}=1 / R, Y_{2}=1 / R+s \quad C_{1}, Y_{4}=1 R+s \quad C_{:}, R=10 \mathrm{~K} \Omega, C_{y} \cong 3 \mathrm{pF}$, $C_{z} \cong 4.5 \mathrm{pF} f=20 \mathrm{MHz}$. 
capacitances $C_{y} \cong 3 \mathrm{pF}$ and $C_{z} \cong 4.5 \mathrm{pF}$ at terminals $y$ and $z$ of the CFOA were exploited to advantage. It appears that the experimental results are in excellent agreement with the theory presented.

\section{CONCLUSION}

New CFOA-based crystal oscillators have been presented. Each circuit uses one CFOA, a crystal and, at most, five externally connected passive elements. In both circuits the output can be taken from a low impedance outlet, thus avoiding the degradation in the crystal performance resulting from external loading effects. The circuit of Figure 1(b) is a "single-pin" oscillator and, therefore, may be more attractive than that of Figure 1(a).

\section{References}

[1] Matthys, R. J., Crystal Oscillator Circuits, New York: Wiley, 1983.

[2] Nordholt, E. H. and Boon, C. A. M. (1990). "Single-Pin" integrated crystal oscillators, IEEE Transactions on Circuits and Systems, 37, 175-182.

[3] Ivanisevic, I. I. and Vasiljevic, D. M. (1993). The quartz crystal oscillator realization using current conveyors, IEEE Transactions on Circuits and Systems-I: Fundamental Theory and Applications, 40, 530-533.

[4] Stiurca, D. (1996). Crystal oscillator using a current-conveyor, Electronics World, $102,324$.

[5] Popovic, J., Pavasovic, A., Zivkovic-DZunja, Z. and Vasiljevic, D. (1996). CMOS RLC and crystal oscillators based on current conveyors, IEEE Transactions on Ultrasonic, Ferroelectrics, and Frequency Control, 43, 410-416.

[6] Akeberg, D. and Mossberg, K. (1974). A versatile active RC building block with inherent compensation for the finite bandwidth of the amplifier, IEEE Transactions on Circuits and Systems, CAS-21, 75-78. 

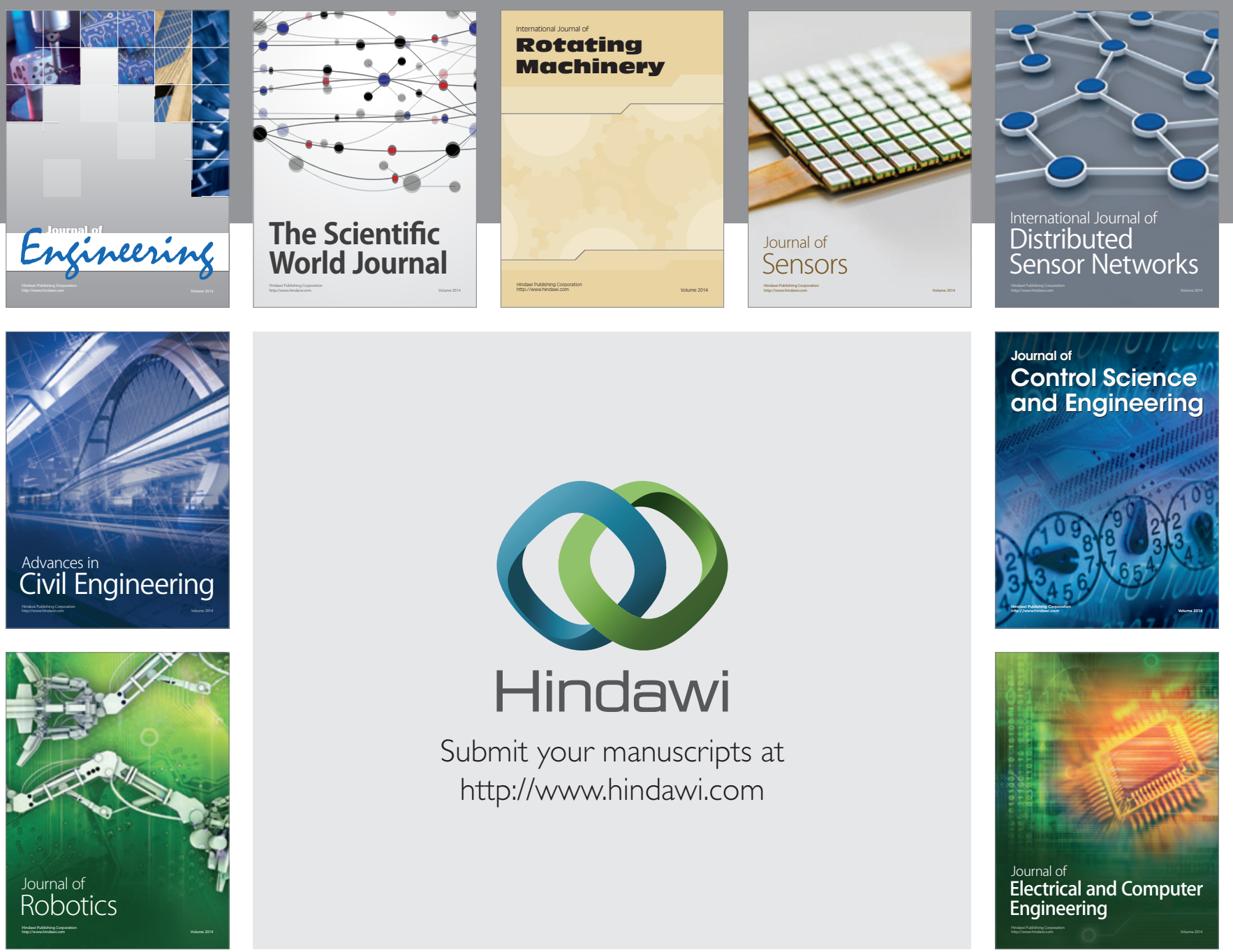

Submit your manuscripts at

http://www.hindawi.com
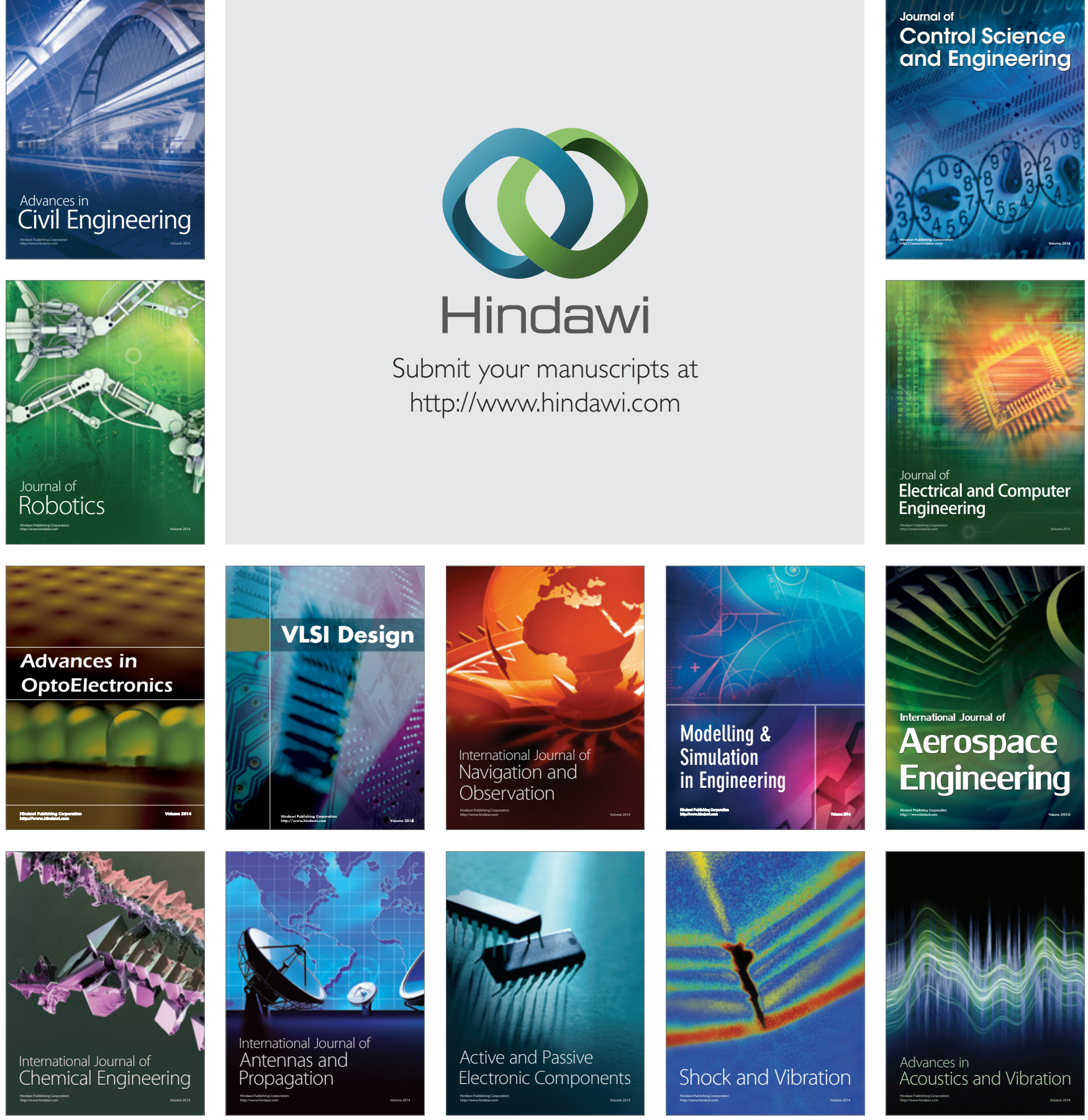